\title{
Un nuevo método no invasivo en la valoración integral de la fibrilación auricular por tomografía cardíaca
}

\author{
A novel, non-invasive, integral imaging assessment in atrial fibrillation by cardiac \\ tomography
}

\author{
Martha Morelos-Guzmán 1,2,", Larissa Minero-García ${ }^{1,4 *, \eta}$, José E. Jaramillo-Almaguer', \\ José F. Chávez-Carbajaß ${ }^{3}$, Carlos A. Arean-Martínez ${ }^{2}$, Juan M. Vargas-Espinosa², \\ Martha E. Viveros-Sandoval${ }^{4}$ e Israel D. Campos-González ${ }^{5,6}$ \\ ${ }^{1}$ Departamento de Imagen Cardiovascular; ${ }^{2}$ Servicio de Medicina Interna y Cardiología; ${ }^{3}$ Centro de Investigación "Mario Alvizouri", Hospital General \\ "Dr. Miguel Silva"; "Facultad de Ciencias Médicas y Biológicas "Dr. Ignacio Chávez", UMSNH, Departamento de Investigación Científica; \\ ${ }^{5}$ Departamento de Investigación y Educación, Clínica de Hemodiálisis NausLife; ${ }^{6}$ Departamento de Investigación de Nefrología, Hospital General \\ "Dr. Miguel Silva". Morelia, Michoacán, México \\ "Martha Morelos-Guzmán y Larissa Minero-García contribuyeron de manera igualitaria en esta investigación.
}

\section{Resumen}

Objetivo: Analizar la utilidad clínica y la viabilidad de la tomografía cardíaca multidetector (TCMD) en la valoración de pacientes con fibrilación auricular (FA). Material y métodos: Estudio prospectivo de casos y controles en 84 sujetos (54 con FA y 30 controles). La morfología de la orejuela izquierda (OI) se clasificó en cactus, ala de pollo, manga de viento y coliflor. La presencia de trombo en la Ol, el antecedente de accidente cerebrovascular y la escala $\mathrm{CHA}_{2} D S_{2}$-VASc se compararon con parámetros obtenidos por TCMD. Resultados: La fracción de expulsión de la aurícula izquierda (FEAl) y la fracción de expulsión de la orejuela izquierda (FEOI) fueron más bajas en los individuos con FA ( $p<0.001)$. El volumen indexado de la aurícula izquierda (VIAl) fue mayor en los pacientes con FA $(p<0.001)$. Se observó una correlación inversa entre la FEAI y el VIAI ( $r=-0.38, p<0.001)$. La morfología tipo coliflor fue la más frecuente en enfermos con FA, mientras que la de tipo cactus predominó en los controles. La de tipo coliflor fue la más relacionada con presencia de trombos $(p<0.01)$, así como una calificación $\mathrm{CHA}_{2} D S_{2}-V A S c$ alta. La velocidad de flujo fue menor en los pacientes con FA ( $\left.p<0.001\right)$. Conclusión: La TCMD es un método novedoso y no invasivo para una valoración integral en la FA. Los resultados de este estudio podrían mejorar la precisión, la utilidad clínica y el análisis de estratificación del riesgo en la FA. Los autores proponen incluir este nuevo método en la valoración integral del riesgo tromboembólico en pacientes con FA.

Palabras clave: Tomografía cardíaca multidetector. Fibrilación auricular. Función auricular. Orejuela izquierda. Velocidad de flujo

\section{Abstract}

Objective: To analyze the clinical utility and feasibility of the multidetector cardiac tomography (MDCT) in multi-parametric imaging assessment in atrial fibrillation (AF) patients. Material and methods: Prospective case-control study in 84 subjects (54 AF subjects and 30 healthy subjects). Left atrial appendage (LAA) morphology was classified as: cactus, chicken wing,

Correspondencia:

*Larissa Minero-García

E-mail: larissamg.gm@ hotmail.com
Disponible en internet: $18-06-2020$ Arch Cardiol Mex. 2021;91(1):42-49 www.archivoscardiologia.com 1405-9940/C 2020 Instituto Nacional de Cardiología Ignacio Chávez. Publicado por Permanyer. Este es un artículo open access bajo la licencia CC BY-NC-ND (http://creativecommons.org/licenses/by-nc-nd/4.0/). 
wind sock, cauliflower. Intra-cardiac thrombus, stroke history and $\mathrm{CHA}_{2} D S_{2}-\mathrm{VASC}$ scale were compared to cardiac MDCT atrial imaging assessment. Results: Left atrial ejection fraction (LAEF) and LAA ejection fraction (LAAEF) were lower in AF subjects $(p<0.001)$, left atrial volume index (LAVI) was higher in AF subjects $(p<0.001)$. An inverse correlation between $L A E F$ and $L A V I$ was found $(r=-0.38, p<0.001)$. Cauliflower $L A A$ morphology frequency was higher in AF subjects, whereas cactus LAA morphology frequency was higher in controls. Cauliflower LAA morphology was associated with thrombus presence $(p<0.01)$ as well as a higher $C H A_{2} D S_{2}-V A S c$ score. Flow velocity were lower in AF subject compared to controls ( $p$ $<0.001)$. Conclusion: MDCT is a novel, non-invasive, worldwide available method for an integral assessment in AF. Our results could improve precision, clinical utility and risk stratification analysis in AF. Our proposal is to include this new method into the global cardiovascular and thrombotic risk assessment in AF patients.

Key words: Multidetector cardiac tomography. Atrial fibrillation. Atrial function. Left atrial appendage. Flow velocity

\section{Introducción}

La fibrilación auricular (FA) es la arritmia cardíaca más común del adulto en la práctica clínica. En la actualidad se calcula una incidencia global de 1 a $2 \%$ en la población general, cerca de 33.5 millones de personas ${ }^{1}$. Alrededor de 1 de cada 5 accidentes cerebrovasculares se debe a FA, casi siempre éstos son graves y producen discapacidad a largo plazo o muerte ${ }^{2}$.

La clasificación $\mathrm{CHA}_{2} \mathrm{DS}_{2}$-VASc es una herramienta utilizada de forma amplia para valorar el riesgo de sufrir un episodio embólico y es esencial para identificar a los pacientes que se benefician del tratamiento anticoagulante ${ }^{3}$. El ecocardiograma transtorácico constituye una herramienta útil y es sin duda la técnica básica de valoración en los pacientes con FA; sin embargo, tiene una capacidad limitada para la detección de trombos en la aurícula izquierda (Al) y la orejuela izquierda $(\mathrm{OI})^{4}$. El ecocardiograma transesofágico (ETE) es la modalidad de imagen más empleada en pacientes con FA antes de procedimientos de ablación de venas pulmonares 0 en el cierre percutáneo de la $\mathrm{Ol}^{5}$. En fecha reciente, Nucifora, et al. han propuesto la ecocardiografía tridimensional para la valoración de la morfología de la $\mathrm{Ol}^{6}$.

La resonancia magnética $(\mathrm{RM})$ cardíaca es una modalidad de imagen no invasiva alternativa en los casos en los que la ETE no es factible, pero su costo elevado y escasa disponibilidad dificultan su uso?.

Los avances actuales en la tomografía cardíaca multidetector (TCMD) permiten una gran resolución espacial y temporal, con imágenes tridimensionales que hacen posible una valoración no invasiva cuantitativa y multiparamétrica de la aurícula y la orejuela izquierdas. Ya existen estudios comparativos entre ETE y TCMD en la identificación de trombos intracavitarios, en los que la TCMD mostró mejor precisión diagnóstica. No obstante, no se ha realizado la comparación de estos dos métodos en pacientes con $\mathrm{FA}^{8}$. El volumen indexado de la aurícula izquierda (VIAI) y la fracción de expulsión de la aurícula izquierda (FEAl) ya se han valorado en pacientes con afección coronaria por $\mathrm{TCMD}^{9}$ y en la población sana ${ }^{10}$. Burrell, et al. encontraron un incremento del VIAI en los pacientes con FA en relación con un grupo control $(p<0.001)$. Además, señalaron que ante la presencia de FA hay una disminución de la contractilidad y la función de la Ol que se manifiesta en la forma de una disminución de las velocidades de flujo y dilatación de la Ol'11. Mügge, et al. demostraron que existe correlación entre la disminución de la fracción de expulsión de la orejuela izquierda (FEOI) y la formación de trombos mediante ETE en pacientes con $\mathrm{FA}^{12}$. Di Biase, et al. clasificaron en cuatro categorías la morfología de la OI: "ala de pollo", que es la más común en la población sana $(48 \%)$, seguida por la de "cactus" (30\%), "manga de viento" (19\%) y "coliflor" $(3 \%)^{13}$. Bosi, et al. demostraron en modelos anatómicos in vitro que la morfología de la Ol se relaciona con la hemodinamia del flujo sanguíneo y sugirieron que a menor velocidad de flujo, mayor posibilidad de episodios trombóticos. Estos especialistas encontraron que la morfología en "coliflor" posee la velocidad de flujo más baja y la de tipo "manga de viento" se relaciona con una velocidad de flujo mayor ${ }^{14}$.

Hoy en día, el método más utilizado para cuantificar la velocidad de flujo de la Ol es el ETE, si bien este método es invasivo y conlleva ciertas complicaciones. Yasuoka, et al. condujeron un estudio en el que correlacionaron la velocidad de flujo de la OI obtenida por ETE y TCMD, y encontraron buena correlación entre ambos métodos ${ }^{15}$. Un metaanálisis reciente que incluyó 19 estudios con 2,955 pacientes mostró una sensibilidad del $96 \%$, una especificidad del $92 \%$ y un sólido valor predictivo negativo de la TCMD para la detección de trombos (96 a 100\%) ${ }^{16}$. Wu, et al. propusieron que los pacientes sin evidencia de trombos por TCMD no requieren ETE para su confirmación ${ }^{17}$. Además, la TCMD ha demostrado en fecha reciente ser superior 


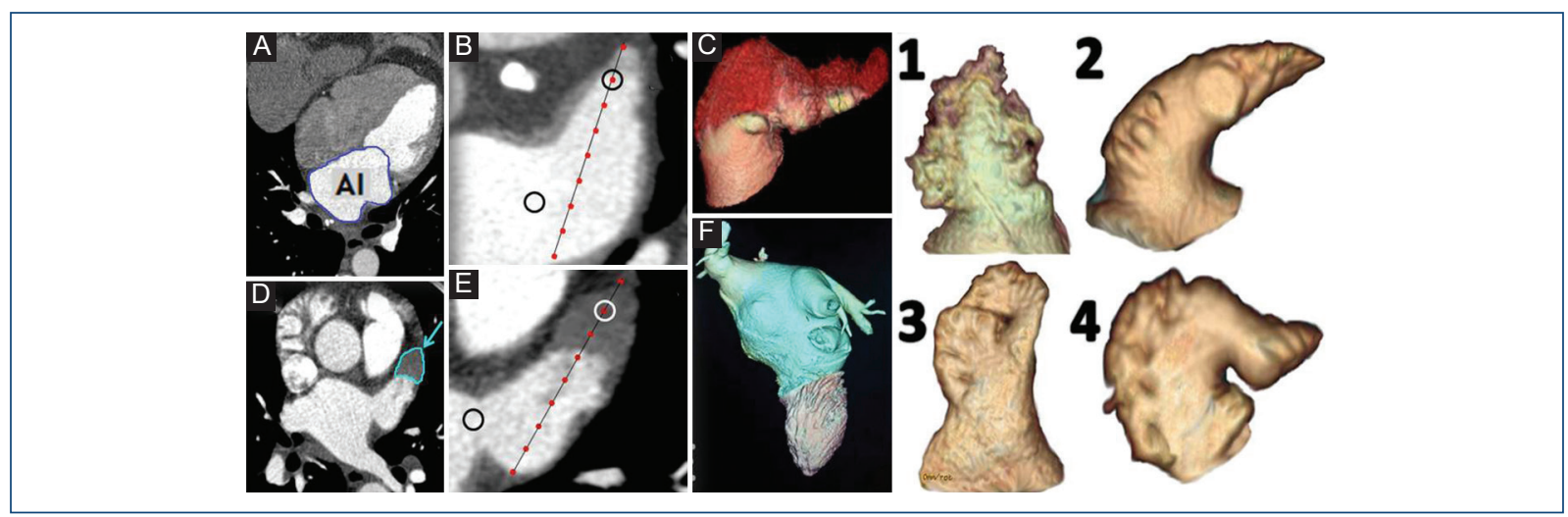

Figura 1. Parámetros por tomografía cardíaca multidetector. A: Volumen indexado de la aurícula izquierda (Al). B: Velocidad de flujo de la orejuela izquierda (OI) en un paciente del grupo control. C: Reconstrucción tridimensional del trombo en la OI. D: Trombo en la Ol (flecha). E: Velocidad de flujo de la Ol en un paciente del grupo de fibrilación auricular. F: Reconstrucción tridimensional de Al aneurísmica. Reconstrucción tridimensional de los diferentes tipos de morfología de Ol: 1) coliflor, 2) manga de viento, 3) cactus, 4) ala de pollo.

al ecocardiograma transtorácico y transesofágico en cuanto a la valoración del tamaño de la $\mathrm{Al}$, un importante predictor de recurrencia de la $\mathrm{FA}^{18}$.

La TCMD posibilita una valoración completa en los pacientes con $\mathrm{FA}$; hasta el momento no existen estudios que evalúen de modo simultáneo la FEAl, la FEOI, el volumen indexado de la Al y OI, la morfología de la OI, la velocidad de flujo de la OI y la presencia de trombo en la OI por TCMD. Esta valoración multiparamétrica ofrece mayor información al médico para optimizar el tratamiento y permitir una reestratificación del riesgo tromboembólico de los pacientes con FA, lo que podría modificar la calidad de vida y el pronóstico de estos pacientes. Este estudio abrirá nuevos horizontes en la investigación relacionada con este tema, ya que las publicaciones médicas son escasas y es previsible que sirva de referencia para futuras investigaciones.

\section{Material y métodos}

Estudio prospectivo comprendido entre el 1 de marzo del 2018 y el 1 de abril del 2019 realizado en el Hospital General "Dr. Miguel Silva" de la ciudad de Morelia, Michoacán, México. El estudio recibió aprobación del comité de ética con número de registro Conbioética-16-CEI-004-20161212, en el cual se incluyó a un total de 84 pacientes. De ellos, 54 tenían diagnóstico de FA y 30 eran del grupo de control. Este último grupo se integró con pacientes sometidos a TCMD por sospecha clínica de cardiopatía isquémica y no padecían FA; estos controles presentaban algún factor de riesgo (hipertensión arterial, diabetes mellitus, dislipidemia, tabaquismo, alcoholismo u obesidad).
Ambos grupos se parearon por edad y género. En todos los pacientes se evaluó la escala de $\mathrm{CHA}_{2} \mathrm{DS}_{2}$-VASc para calcular el riesgo tromboembólico. Se incluyeron el peso y la talla para calcular el índice de masa corporal (IMC), así como otras variables clínicas como tabaquismo, alcoholismo, hipertensión arterial (HAS), diabetes mellitus (DM), dislipidemia y antecedente de episodio vascular cerebral o ictus transitorio (EVC/IT). Se excluyó a los pacientes con enfermedad valvular, miocardiopatías, enfermedades congénitas, enfermedades autoinmunitarias, insuficiencia renal o alergia al medio de contraste. Todos los pacientes se sometieron a TCMD para obtener las imágenes tal y como lo describieron Gweon, et al. ${ }^{19} \mathrm{Se}$ utilizaron tomógrafos de 32 y 64 cortes (General Electric) y se administró medio de contraste (lopamiron $® 370$ ). Se administró betabloqueador con metoprolol por vía oral antes del estudio con el objetivo de lograr una frecuencia cardíaca de 60 latidos/min. Las estructuras tridimensionales de la $\mathrm{Al}$ y Ol se reconstruyeron con la técnica de procesamiento de volumen rendering para valorar el volumen indexado y la fracción de expulsión de la Al y OI. Se calcularon las variables velocidad de flujo y volumen de la Al y OI, así como la presencia de trombos. La morfología de la Ol se evalúo en reconstrucciones multiplanares y la clasificaron dos expertos en imagen cardíaca, que estaban cegados a los datos clínicos de los pacientes.

Un especialista en imágenes cardiovasculares efectuó el análisis de las imágenes obtenidas mediante una estación de trabajo GE (CardiolQ Express, versión 8; General Electric Medical Systems). Para determinar la velocidad de flujo de la Ol se obtuvo una imagen bidimensional de dicha estructura y con posterioridad se 
Tabla 1. Características clínicas del grupo de control y el grupo de fibrilación auricular

\begin{tabular}{|c|c|c|c|}
\hline & $\begin{array}{l}\text { Grupo de } \\
\text { control } \\
(n=30)\end{array}$ & $\begin{array}{c}\text { Grupo FA } \\
(n=54)\end{array}$ & p \\
\hline Edad (años) & $54.83 \pm 15$ & $\begin{array}{c}67.43 \pm \\
14\end{array}$ & $<0.001$ \\
\hline Género masculino & $53.3 \%$ & $55.6 \%$ & $\mathrm{~N} / \mathrm{S}$ \\
\hline IMC & $27.9 \pm 5$ & $28 \pm 5$ & $\mathrm{~N} / \mathrm{S}$ \\
\hline Hipertensión arterial & $43.3 \%$ & $46.3 \%$ & $\mathrm{~N} / \mathrm{S}$ \\
\hline Dislipidemia & $23.3 \%$ & $24.1 \%$ & $\mathrm{~N} / \mathrm{S}$ \\
\hline Diabetes mellitus & $6.7 \%$ & $25.9 \%$ & $<0.001$ \\
\hline Tabaquismo & $36.7 \%$ & $31.5 \%$ & $\mathrm{~N} / \mathrm{S}$ \\
\hline Alcoholismo & $23.3 \%$ & $22.2 \%$ & $\mathrm{~N} / \mathrm{S}$ \\
\hline Antecedente de EVC/IT & $0 \%$ & $33.3 \%$ & $<0.001$ \\
\hline Anticoagulación & $0 \%$ & $42.6 \%$ & $<0.001$ \\
\hline $\begin{array}{l}\text { Antiagregación } \\
\text { plaquetaria }\end{array}$ & $0 \%$ & $53.7 \%$ & $<0.001$ \\
\hline $\begin{array}{l}\text { Tipo de FA } \\
\text { FA paroxística } \\
\text { FA permanente }\end{array}$ & & $\begin{array}{l}38.9 \% \\
61.1 \%\end{array}$ & \\
\hline Escala $\mathrm{CHADS}_{2}$ & & $1.7 \pm 1.2$ & \\
\hline Escala $\mathrm{CHA}_{2} \mathrm{DS}_{2}$-VASc & & $2.8 \pm 1.7$ & \\
\hline
\end{tabular}

IMC: índice de masa corporal; EVC: episodio vascular cerebral; IT: ictus transitorio; FA: fibrilación auricular.

determinaron dos puntos distintos en la OI (distal y proximal) (Fig. 1, B y E); el punto proximal se obtuvo en el centro del orificio de la Ol, mientras que el punto distal fue el punto $7 / 8$ de una línea recta trazada desde el punto de flexión de la pared posterior de la OI hasta el punto distal más anterior de la OI. Una vez conseguidos estos dos valores, se llevó a cabo una relación del punto distal sobre el punto proximal, y el resultado fue una relación de unidades Hounsfield. Yasuoka describió y aprobó con anterioridad este método ${ }^{15}$, la morfología de la Ol se evaluó de acuerdo con la clasificación de Di Biase ${ }^{13}$ y el volumen indexado de la Ol se calculó a partir de los volúmenes máximo y mínimo de la Ol que se determinaron con la técnica de procesamiento de volumen rendering. La FEOI se calculó con la siguiente fórmula: $E F=\left(V_{\text {máx }}\right.$ $\left.\left.-\mathrm{V}_{\text {min }}\right) / V_{\text {máx }}\right)$, con base en el método de Boucebci ${ }^{20}$.

\section{Análisis estadístico}

Se aplicó un análisis de varianza de una vía o bien $\mathrm{H}$ de Kruskal-Wallis con post hoc de Dunn en variables continuas, como edad, peso, índice de masa corporal, y todas las características cuantitativas radiológicas agrupadas por el tipo de Ol, tanto en el grupo de FA como en los controles; se aplicó contraste estadístico de promedios entre la FA y los controles y, dentro del grupo de FA, entre los dos subgrupos de FA (paroxística vs. permanente) con t de Student con una y dos colas, respectivamente; se analizó el coeficiente de correlación lineal producto del momento de Pearson para los dos grupos en conjunto con la velocidad de flujo de Ol respecto de las demás variables cuantitativas y se aplicaron las pruebas de $\chi^{2}$ o $\mathrm{F}$ exacta de Fisher para el contraste de frecuencias en cuadrado latino de $2 \times 2$; en todos los casos, la probabilidad estadísticamente significativa del error alfa fue $<0.05$, según el programa de SigmaPlot 12.5 (CR) 2003-2013.

\section{Resultados}

Se analizó a 84 individuos, 54 de ellos con FA: $21(38.9 \%)$ con FA paroxística y $33(61.1 \%)$ con FA permanente. La edad media en el grupo de FA fue de $67 \pm 14$ años y $55.6 \%$ correspondía a hombres. La tabla 1 describe las características clínicas de ambos grupos. Se encontró una correlación inversa entre la FEAl y la edad $(r=-0.43, p<0.001)$, al igual que con la FEOI $(r=-0.33, p<0.001)$.

La morfología de tipo coliflor fue la más frecuente en el grupo de FA (33.3\%), mientras que la de tipo cactus predominó en los controles (37\%). En el análisis del VIAI y la FEAI se encontraron diferencias significativas entre ambos grupos $(p<0.001)$, y asimismo se documentaron diferencias con la FEOI y la velocidad de flujo de la Ol entre casos y controles $(p<0.001)$.

Los parámetros evaluados por TCMD se consignan en la tabla 2. La velocidad de flujo de la OI fue más baja en el grupo de FA respecto del grupo control $(0.34 \pm$ $0.16 \mathrm{~cm} / \mathrm{s}$ vs. $0.87 \pm 0.08 \mathrm{~cm} / \mathrm{s})(p<0.001)$. En los pacientes con FA, la velocidad de flujo de la Ol fue más baja en quienes tuvieron trombo en la Ol, pero no se encontró diferencia estadística en relación con los que no sufrieron trombo $(0.32 \pm 0.14$ vs. $0.35 \pm 0.17 \mathrm{~cm} / \mathrm{s} ; p=N S)$ (Fig. 2).

En el análisis del grupo de FA se observó que el VIAl fue mayor en los pacientes que presentaron trombo en la Ol en relación con los que no lo tuvieron $\left(82 \pm 32.7\right.$ vs. $48.3 \pm 14.9 \mathrm{ml} / \mathrm{m}^{2}$ respectivamente; $p<0.001)$. La FEAl fue menor en quienes mostraron trombo en la Ol respecto de los individuos sin él (14.1 \pm 7.9 vs. $23.6 \pm 11.8 \% ; p<0.001$ ); los mismos resultados se obtuvieron con la FEOI $(11.6 \pm 6.2$ vs. $18.2 \pm 9 \% ; p=0.002$ ) (Fig. 3). 
Tabla 2. Variables por tomografía cardíaca multidetector

\begin{tabular}{|c|c|c|c|}
\hline & $\begin{array}{l}\text { Grupo } \\
\text { control } \\
(n=30)\end{array}$ & $\begin{array}{l}\text { Grupo FA } \\
(n=54)\end{array}$ & p \\
\hline $\begin{array}{l}\text { Calificación de calcio } \\
\text { (UA) }\end{array}$ & $10.8 \pm 24$ & $59.26 \pm 142$ & $\mathrm{~N} / \mathrm{S}$ \\
\hline VIAI $\left(\mathrm{ml} / \mathrm{m}^{2}\right)$ & $40.3 \pm 9$ & $67.3 \pm 27.4$ & $<0.001$ \\
\hline $\mathrm{Vol}_{\text {máx }} \mathrm{Al}(\mathrm{ml})$ & $75.2 \pm 20$ & $116.4 \pm 49$ & $<0.001$ \\
\hline $\mathrm{Vol}_{\text {min }} \mathrm{Al}$ (ml) & $53.4 \pm 19.3$ & $96.6 \pm 42.8$ & $<0.001$ \\
\hline FEAI (\%) & $29.8 \pm 12.5$ & $16.2 \pm 7.7$ & $<0.001$ \\
\hline $\mathrm{Vol}_{\text {máx }}$ 이 (ml) & $10.8 \pm 4.4$ & $12.6 \pm 5.7$ & $\mathrm{~N} / \mathrm{S}$ \\
\hline $\mathrm{Vol}_{\text {mín }}$ Ol (ml) & $8.5 \pm 3.7$ & $11 \pm 5.4$ & $<0.001$ \\
\hline FEOI (\%) & $21.1 \pm 9$ & $13.87 \pm 7.7$ & $<0.001$ \\
\hline VFOI (cociente UH)> & $0.72 \pm 0.20$ & $0.34 \pm 0.16$ & $<0.001$ \\
\hline $\begin{array}{l}\text { Morfología de la OI } \\
\text { Coliflor } \\
\text { Manga de viento } \\
\text { Ala de pollo } \\
\text { Cactus }\end{array}$ & $\begin{array}{c}20.0 \% \\
26.7 \% \\
16.7 \% \\
37 \%\end{array}$ & $\begin{array}{l}33.3 \% \\
27.8 \% \\
16.7 \% \\
22.2 \%\end{array}$ & $\mathrm{~N} / \mathrm{S}$ \\
\hline Trombo (\%) & $0 \%$ & $42.5 \%$ & $<0.001$ \\
\hline
\end{tabular}

VIAl: volumen indexado de la aurícula izquierda; Vol ${ }_{\text {max }} \mathrm{Al}$ : volumen máximo de la aurícula izquierda; $V_{0} I_{\min }$ Al: volumen mínimo de la aurícula izquierda;

FEAl: fracción expulsión de la aurícula izquierda; $V_{\text {móx }}$ Ol: volumen máximo de la orejuela izquierda; Vol Ol: volumen mínimo de la orejuela izquierda; FEOI: fracción expulsión de la orejuela izquierda; VFOI: velocidad de flujo de la orejuela izquierda.

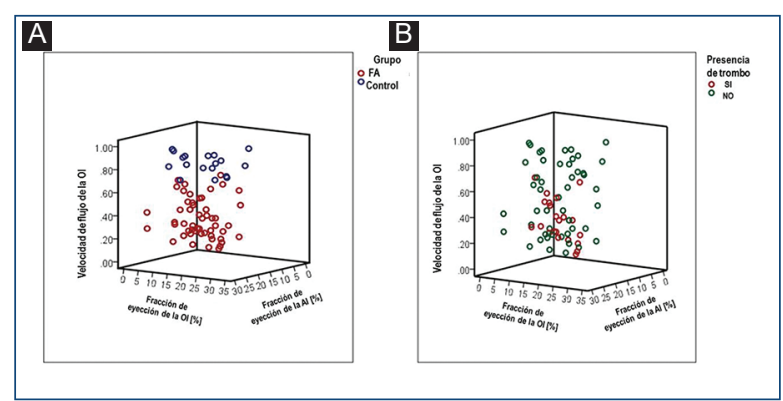

Figura 2. A: Velocidad de flujo de la orejuela izquierda (OI) en sujetos con fibrilación auricular (FA) en comparación con los controles. B: Velocidad de flujo de la Ol en pacientes con FA con y sin trombo. Grupo de FA contra grupo control ( $p<0.01$; prueba T de Student).

Se identificaron algunas diferencias importantes en cuanto a la morfología de la Ol en el grupo de FA; la morfología de tipo coliflor fue la más común en los pacientes en los que se detectó trombo en la OI $(p=<0.01)$; con posterioridad se comparó la morfología de tipo coliflor con las formas distintas de ésta en los pacientes con trombo en la Ol y se observaron 5.9 vs. $7.4 \%(\mathrm{p}<$ 0.001); OR, 4.7; y IC95\%, 1.7 a 12.4, respectivamente. La morfología de tipo manga de viento se vinculó con ausencia de trombo $(p<0.05)$ (Fig. 4). Se comparó una calificación $\mathrm{CHA}_{2} \mathrm{DS}_{2}$-VASc alta respecto de baja a moderada con la presencia de trombo en la OI, con hallazgo de trombo en 16.7 vs. $9.3 \%(p=0.056) ;$ OR, 2.4; IC95\%, 0.93 a 6.2, respectivamente.

En el grupo de FA, 23 pacientes (42.6\%) tenían tratamiento anticoagulante. De estos individuos, $10(43.5 \%)$ presentaron trombo en la Ol, mientras que en los no anticoagulados $13(41.9 \%)$ tuvieron trombo en la OI $(p<0.01)$.

\section{Discusión}

En la actualidad, el ETT y el ETE son las modalidades de imagen más utilizadas para la valoración de estratificación del riesgo en pacientes con FA; sin embargo, este último es un método invasivo que supone riesgos potenciales ${ }^{20}$. El ETE se considera la norma de referen$\mathrm{Cia}^{2}$ en la identificación de trombos en la FA. La TCMD es un método no invasivo que permite una valoración multiparamétrica en pacientes con FA; no obstante, su papel no está bien definido, ya que existen pocos estudios al respecto y, hasta la fecha, ninguna publicación ha evaluado en forma integral a pacientes con FA mediante TCMD. En esta población de estudio se incluyó a un total de 84 pacientes y la media de edad de los enfermos con FA fue de 67 años contra 55 años en el grupo control. A pesar de que no se tuvo el mismo número de muestra para ambos grupos, este parámetro fue significativo $(p<0.001)$ y concuerda con las publicaciones médicas en las que la edad promedio de la FA se halla alrededor de la séptima década de la vida. Esto es importante porque existe un incremento de la FA cuanta mayor sea la edad ${ }^{21}$. Las características basales entre ambos grupos de esta población de estudio (tabaquismo, alcoholismo, hipertensión arterial sistémica y dislipidemia) no mostraron diferencias significativas.

En cuanto al tratamiento de pacientes con FA se identificó que sólo 42.6\% (23 pacientes) se encontraba bajo tratamiento anticoagulante, mientras que 53.7\% (29 pacientes) consumía antiagregantes plaquetarios. Esto probablemente se debía a que un gran porcentaje de estos pacientes acudió por primera vez a la consulta externa de cardiología en la que se estableció el diagnóstico y se inició tratamiento. La dilatación de la Al es un marcador de episodios cardiovasculares adversos, como ictus, insuficiencia cardíaca o incluso la muerte. Pavón, et al. mencionan que el VIAI debe ser una medida sistemática 


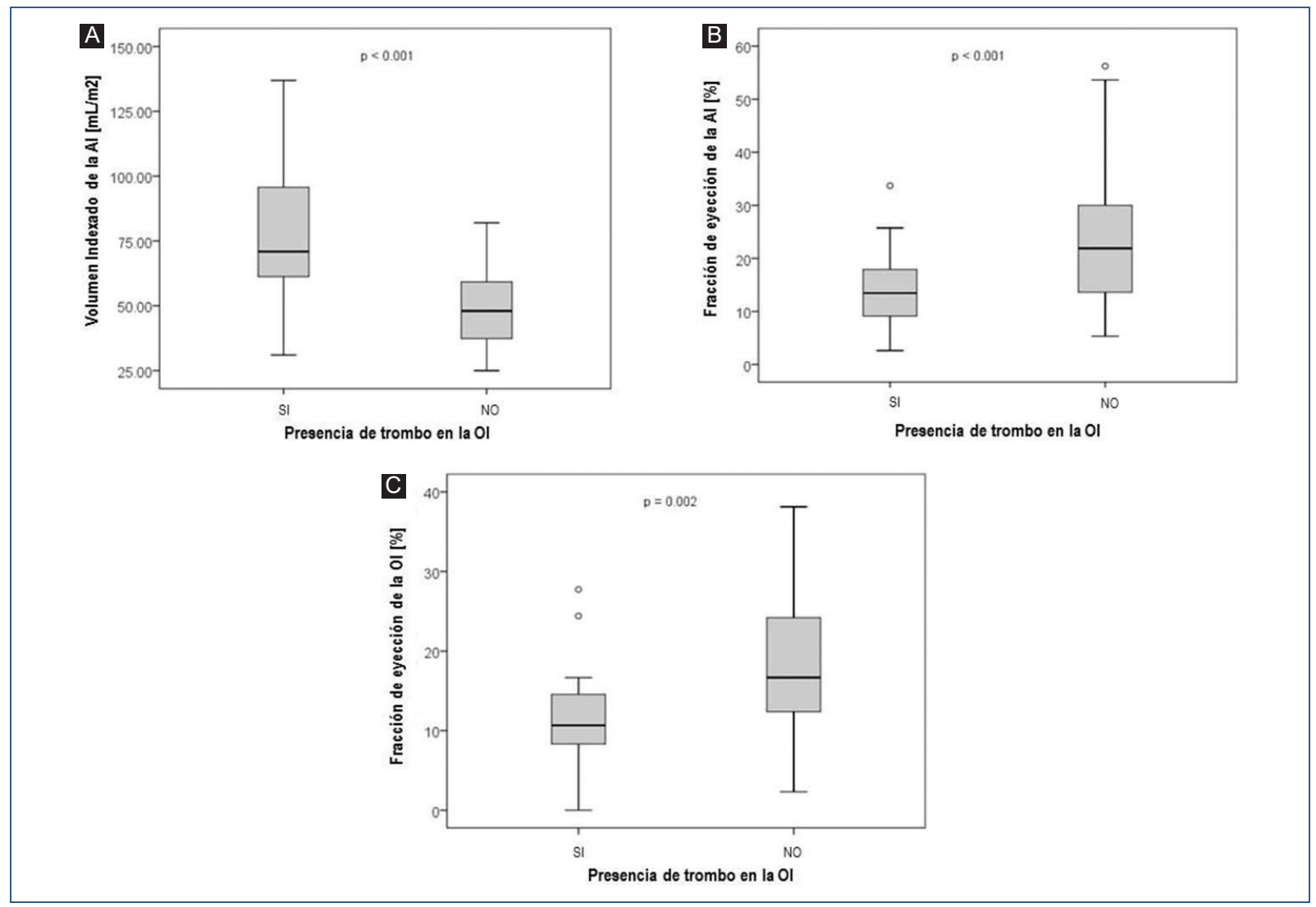

Figura 3. Diferencias entre el volumen indexado de la aurícula izquierda y las fracciones de expulsión de la aurícula y la orejuela izquierda en pacientes con y sin trombo (prueba T de Student).

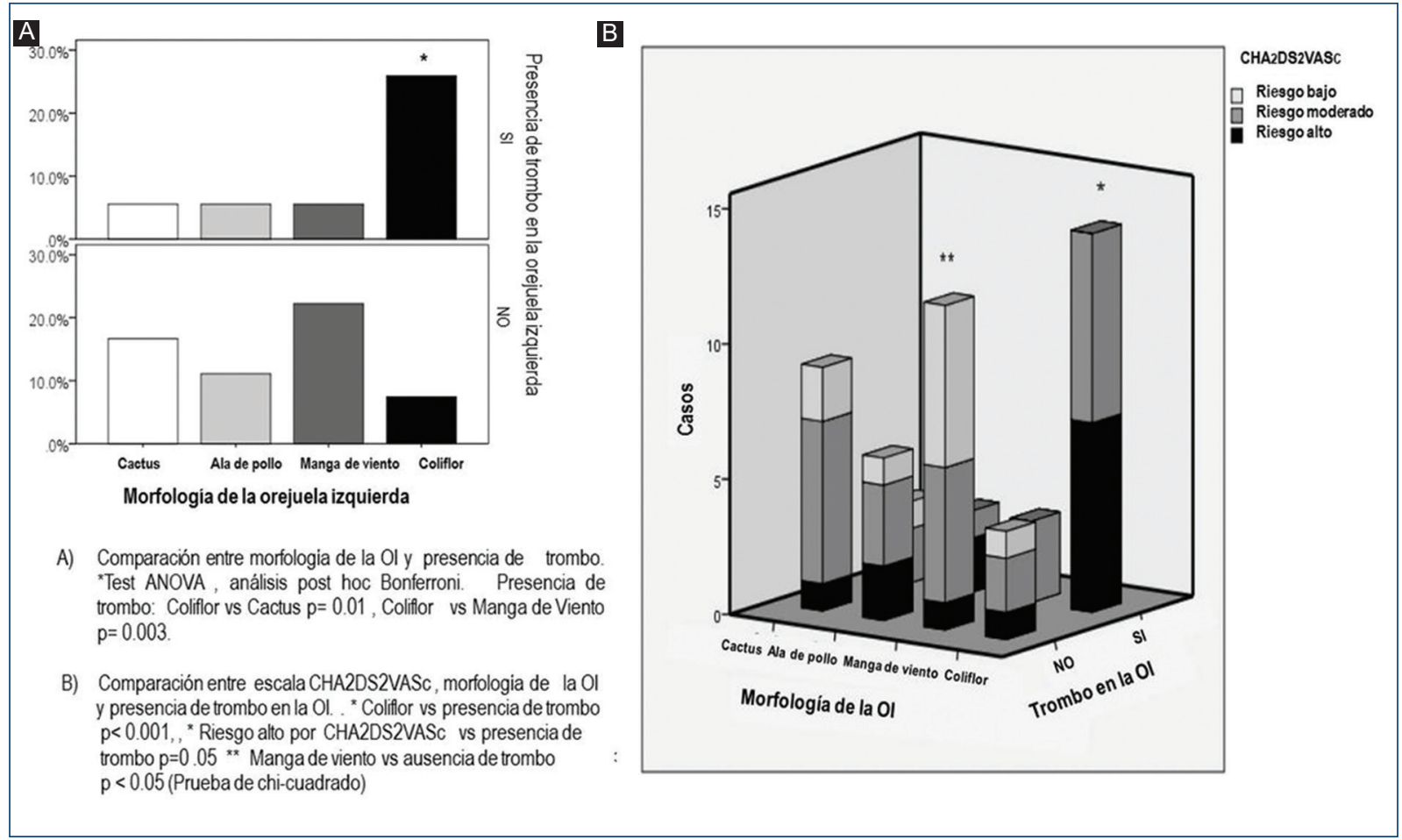

Figura 4. Comparación entre los diferentes tipos de morfología de la orejuela izquierda y la escala $\mathrm{CHA}_{2} \mathrm{DS}_{2}$-VASc. 
de valoración de crecimiento auricular por su alta sensibilidad y especificidad. Además, refleja una elevación de la presión de llenado ventricular izquierdo y por tanto es un importante predictor de mal pronóstico en pacientes con $\mathrm{FA}^{22}$. En este estudio, los autores encontraron que el VIAI fue marcadamente mayor en la población de FA respecto del grupo control, a pesar de que los controles también registraron valores alterados de VIAI $(40.3 \pm$ $\left.9 \mathrm{ml} / \mathrm{m}^{2} ; p<0.001\right)$. Esto es importante puesto que la FA causa per se crecimiento auricular, lo que favorece un aumento de la presión de llenado y con ello se establece un ciclo de autoperpetuación de la arritmia. En consecuencia, la restauración del ritmo sinusal previene y revierte la dilatación auricular en estos pacientes, al normalizar las presiones de llenado y el gasto cardíaco, lo cual mejora el pronóstico del paciente ${ }^{22}$. En cuanto a la morfología de la OI, en este estudio no se identificaron diferencias significativas entre ambos grupos, probablemente debido al número pequeño de la muestra. No obstante, se observó que la morfología en "coliflor" fue la más relacionada con la presencia de trombos en la OI. Se encontró que los tipos en "manga de viento" y "ala de pollo" eran los menos vinculados con la presencia de trombos. Estos resultados coinciden con los de Bosi, et al., quienes encontraron que la Ol de tipo "coliflor" tenía una velocidad de flujo menor que el resto de las morfologías y, si bien éste es un estudio en modelos anatómicos, proporciona un análisis importante sobre la fisiopatología de la trombogénesis en la Ol' ${ }^{14}$. En los pacientes con FA se reconoció una marcada disminución de la velocidad de flujo de la Ol en relación con los controles $(p<0.001)$. Esto puede explicarse por las diferencias anatómicas de cada orejuela (los ángulos que poseen los lóbulos principales respecto de los ostium, el número de trabeculaciones y el número de lóbulos). La forma en "ala de pollo" revela un ángulo obtuso que permite una velocidad de flujo mayor (vaciamiento más rápido); algunos estudios han descrito que es la que menos se relaciona con la presencia de trombos. Las orejuelas de tipo "coliflor" poseen un ángulo agudo respecto del ostium que dificulta su vaciamiento y por ende da lugar a que esta forma se vincule las más de las veces con la formación de trombos ${ }^{23}$. En cuanto a la fracción de expulsión de la Al y OI en la población general, se ha demostrado una correlación inversa (a mayor edad, menor fracción de expulsión); Handke, et al. ${ }^{24}$ encontraron resultados similares. En este estudio se observó que la fracción de expulsión fue menor en el grupo de FA en relación con los controles $(p<0.001) y$, dentro del grupo de FA, la fracción de expulsión fue menor cuando existían trombos en la OI ( $p<0.001)$; esto puede considerarse un criterio de peor pronóstico en este grupo de pacientes. Otra variable clínica analizada fue la valoración de la escala $\mathrm{CHA}_{2} \mathrm{DS}_{2}$-VASc, en la cual se identificó que en la población con FA predominó un valor alto y el tipo que se vinculó con este parámetro fue el de "coliflor". Además, en el grupo de FA, 18 pacientes tenían antecedentes de un episodio vascular (EVC o IT). En este subgrupo de pacientes, el tipo de "coliflor" predominó sobre los demás, lo cual apoya una vez más la teoría de que esta fisonomía de la orejuela es la más trombogénica.

La limitación principal del estudio es la misma que ha enfrentado la mayoría de los autores que han publicado sobre este aspecto, es decir, el tamaño de la muestra. Pese a ello, en esta investigación preliminar los autores registraron resultados significativos. Una de las ventajas para la realización de este estudio fue la integración de un equipo multidisciplinario formado por cardiólogos clínicos, especialistas en imagen cardiovascular y radiólogos.

En la actualidad, el ETE se realiza para descartar la presencia de trombos en la Ol antes de procedimientos invasivos de la FA con fines terapéuticos, como es la oclusión de la orejuela izquierda o la ablación de las venas pulmonares; sin embargo, es importante mencionar que este método es invasivo y existe el riesgo de potenciales complicaciones con el procedimiento, además de un mayor costo en comparación con la $\mathrm{TCMD}^{20}$. Ésta es un método no invasivo que se utiliza hoy en día. Algunas desventajas de este método en relación con los otros son los costos, el uso de medio de contraste y la exposición a la radiación. No obstante, la TCMD tiene a su favor una mejor resolución espacial y temporal respecto de otros métodos, lo cual es de importancia en este estudio para la determinación correcta de la morfología de la orejuela. Además, con los nuevos tomógrafos, la dosis de radiación es cada vez menor (3-8 mSv) ${ }^{25}$.

De este estudio se desprende que los pacientes con FA pueden requerir, junto con una valoración clínica con la escala $\mathrm{CHA}_{2} \mathrm{DS}_{2}-\mathrm{VASc}$, electrocardiograma, vigilancia Holter y ecocardiograma, además de una TCMD, ya que es posible obtener información como la morfología de la OI, parámetros de función auricular y de la Ol, así como la velocidad de flujo, que tienen al parecer un papel importante en la trombogénesis de la OI. Luego de obtener estos parámetros a través de este método es posible seleccionar el tratamiento más conveniente para cada paciente, lo que resulta una aplicación importante y novedosa, y elegir a los enfermos aptos para la oclusión de la orejuela OI. 
Este estudio detalla los hallazgos de forma descriptiva respecto de la TCMD y no fue posible determinar la sensibilidad, la especificidad y la tasa de falsos positivos y negativos dado que no se comparó con la norma de referencia actual, esto es, el ETE. Es importante comparar dichos métodos en futuras investigaciones y determinar estos parámetros, ya que las publicaciones son escasas acerca de este tema.

\section{Conclusiones}

La TCMD es un método no invasivo que permite una valoración integral, útil y precisa en la reestratificación del riesgo tromboembólico en pacientes con FA y proporciona información relevante para tomar decisiones en el tratamiento y el pronóstico de los pacientes con FA. Este protocolo debe implementarse en el abordaje de estudio de los pacientes con FA.

\section{Financiamiento}

Esta investigación no recibió ninguna subvención específica de agencias de financiamiento en los sectores público, comercial o con fines de lucro.

\section{Conflicto de intereses}

Los autores declaran no tener conflicto de intereses.

\section{Responsabilidades éticas}

Protección de personas y animales. Los autores declaran que los procedimientos seguidos se adecuan a las normas éticas del comité de experimentación humana responsable y a la Asociación Médica Mundial y la Declaración de Helsinki.

Confidencialidad de los datos. Los autores declaran que han seguido los protocolos de su centro de trabajo sobre la publicación de datos de pacientes.

Derecho a la privacidad y consentimiento informado. Los autores han obtenido el consentimiento informado de los pacientes o sujetos referidos en el artículo. Este documento obra en poder del autor de correspondencia.

\section{Bibliografía}

1. Morin DP, Bernard ML, Madias C, Rogers PA, Thihalolipavan S, Estes NAM. The state of the art. Mayo Clin Proc. 2016;91(12):1778-810.

2. National Collaborating Centre for Chronic Conditions (UK). Chronic heart failure: National Clinical Guideline for Diagnosis and Management in Primary and Secondary Care. London: Royal College of Physicians (UK) 2003 (National Institute for Health and Clinical Excellence: Guidance). Disponible en: http://www.ncbi.nlm.nih.gov/books/NBK65609
3. Coppens M, Eikelboom JW, Hart RG, Yusuf S, Lip GYH, Dorian P, et al The CHA2DS2-VASc score identifies those patients with atrial fibrillation and a CHADS2 score of 1 who are unlikely to benefit from oral anticoagulant therapy. Eur Heart J. 2013;34(3):170-6.

4. Aschenberg W, Schlüter M, Kremer P, Schröder E, Siglow V, Bleifeld W. Transesophageal two-dimensional echocardiography for the detection of left atrial appendage thrombus. J Am Coll Cardiol. 1986;7(1):163-6.

5. Sallach JA, Puwanant S, Drinko JK, Jaffer S, Donal E, Thambidorai SK, et al. Comprehensive left atrial appendage optimization of thrombus using surface echocardiography: The CLOTS Multicenter Pilot Trial. J Am Soc Echocardiogr. 2009;22(10):1165-72.

6. Nucifora G, Faletra FF, Regoli F, Pasotti E, Pedrazzini G, Moccetti T, et al. Evaluation of the Left atrial appendage with real-time 3-dimensional transesophageal echocardiography: implications for catheter-based left atrial appendage closure. Circulation Cardiovasc Imaging. 2011;4(5):514-23.

7. Rathi VK, Reddy ST, Anreddy S, Belden W, Yamrozik JA, Williams RB, et al. Contrast-enhanced CMR is equally effective as TEE in the evaluation of left atrial appendage thrombus in patients with atrial fibrillation undergoing pulmonary vein isolation procedure. Heart Rhythm. 2013;10(7):1021-7.

8. Vázquez de Prada JA, Pérez de Isla L. Técnicas de imagen en la fibrilación auricular. Rev Esp Cardiol Supl. 2016;16:20-4.

9. Stojanovska J, Cronin P, Patel S, Gross BH, Oral H, Chughtai K, et al. Reference normal absolute and indexed values from ecg-gated mdct: left atrial volume, function, and diameter. Am J Roentgenol. 2011;197(3):631-7.

10. 49: using 64-slice mdct to assess right atrial appendage anatomy and function at sinus rhythm and atrial fibrillation: a comparative study with the left atrial appendage and thrombus formation implications. J Cardiovasc Comp Tomograp. 2008;2:S18.

11. Burrell LD, Horne BD, Anderson JL, Muhlestein JB, Whisenant BK. Usefulness of left atrial appendage volume as a predictor of embolic stroke in patients with atrial fibrillation. Am J Cardiol. 2013;112(8):1148-52.

12. Mügge A, Kühn H, Nikutta $P$, Grote J, G. López JA, Daniel WG. Assessment of left atrial appendage function by biplane transesophageal echocardiography in patients with nonrheumatic atrial fibrillation: identification of a subgroup of patients at increased embolic risk. J Am Coll Cardiol. 1994;23(3):599-607.

13. Di Biase L, Santangeli P, Anselmino M, Mohanty P, Salvetti I, Gili S, et al. Does the left atrial appendage morphology correlate with the risk of stroke in patients with atrial fibrillation? J Am Coll Cardiol. 2012;60(6):531-8.

14. Bosi GM, Cook A, Rai R, Menezes LJ, Schievano S, Torii R, et al. Computational fluid dynamic analysis of the left atrial appendage to predict thrombosis risk. Front Cardiovasc Med. 2018;5:34.

15. Yasuoka R, Kurita T, Kotake Y, Akaiwa Y, Hashiguchi N, Motoki K, et al. A novel method to estimate blood flow velocity in the left atrial appendage using enhanced computed tomography: role of Hounsfield unit density ratio at two distinct points within the left atrial appendage. Heart Vessels. 2017;32(7):893-901.

16. Romero J, Husain SA, Kelesidis I, Sanz J, Medina HM, García MJ. Detection of left atrial appendage thrombus by cardiac computed tomography in patients with atrial fibrillation: a meta-analysis. Circ Cardiovasc Imaging. 2013;6(2):185-94.

17. Wu X, Wang C, Zhang C, Zhang Y, Ding F, Yan J. Systematic review/ meta-analysis computed tomography for detecting left atrial thrombus: a meta-analysis. Arch Med Sci. 2012;6:943-51.

18. Hof I, Arbab口Zadeh A, Scherr D, Chilukuri $K$, Dalal $D$, Abraham $T$, et al. Correlation of left atrial diameter by echocardiography and left atrial volume by computed tomography. J Cardiovasc Electrophysiol. 2009;20(2):159-63.

19. Gweon HM, Kim SJ, Kim TH, Lee SM, Hong YJ, Rim S-J. Evaluation of left atrial volumes using multidetector computed tomography: comparison with echocardiography. Korean J Radiol. 2010;11(3):286.

20. Boucebci S, Pambrun T, Velasco S, Duboe P-O, Ingrand P, Tasu J-P Assessment of normal left atrial appendage anatomy and function over gender and ages by dynamic cardiac CT. Eur Radiol. 2016;26(5):1512-20.

21. Christiaens L, Lequeux B, Ardilouze P, Ragot S, Mergy J, Herpin D, et al. A new method for measurement of left atrial volumes using 64-slice spiral computed tomography: comparison with two-dimensional echocardiographic techniques. Int J Cardiol. 2009;131(2):217-24.

22. Pavón-Jiménez R, García-Medina D, Villagómez-Villegas D. ¿Debemos medir siempre el tamaño de la aurícula izquierda en pacientes con fibrilación auricular? ¿Cómo? ¿Por qué? Cardiocore. 2010;45(3):93-5.

23. Mulder MJ, Götte MJW, Allaart CP. Left atrial appendage morphology in atrial fibrillation: Do we prefer chicken wing or cauliflower? J Cardiovasc Comput Tomogr. 2019;:S1934592519301662.

24. Handke M, Harloff A, Hetzel A, Olschewski M, Bode C, Geibel A. Left atrial appendage flow velocity as a quantitative surrogate parameter for thromboembolic risk: determinants and relationship to spontaneous echocontrast and thrombus formation-a transesophageal echocardiographic study in 500 patients with cerebral ischemia. J Am Soc Echocardiogr. 2005;18(12):1366-72.

25. Izeta-Gutiérrez AC, Ojeda-Delgado JL, Mendizábal-Méndez AL, Valdez-Becerril G, Millán-Contreras S, Gutiérrez-García Z, et al. Tomografía computada de corazón, una herramienta invaluable. Rev Sanid Mil. 2014;68(1):36-41. 